\title{
Rendezvous on a Planar Lattice
}

Steve Alpern ${ }^{1}$ and Vic Baston ${ }^{1,2}$

${ }^{1}$ Department of Mathematics, London School of Economics, Houghton Street, London WC2A 2AE

(s.alpern@lse.ac.uk)

2 Department of Mathematics, University of Southampton, Southampton,Hampshire, SO17 1BJ (vicbaston@bitinternet.com)

Supported by NATO grant PST.CLG.976391

October 2, 2004 


\begin{abstract}
We analyze the optimal behavior of two players who are lost on a planar surface and who want to meet each other in least expected time. They each know the initial distribution of the other's location, but have no common labeling of points, and so cannot simply go to a location agreed to in advance. They have no compasses, so do not even have a common notion of North. For simplicity, we restrict their motions to the integer lattice $Z^{2}$ (graph paper) and their motions to horizontal and vertical directions, as in the original work of Anderson and Fekete.
\end{abstract}

Keywords: rendezvous, search, plane 


\section{Introduction}

This paper considers rendezvous search problems in the plane. Two players, I and II, are initially placed so that the vector from I to II has a known distribution. The players move at unit speed, until the first time $\tau$ that they meet. Both of them wish to minimize the expected value of the meeting time $\tau$. We consider the so called player - asymmetric version of the problem, in which the players may adopt distinct strategies; for example one might stay still while the other one followed an optimal exhaustive search (this is called the Wait For Mommy strategy). We follow the approach introduced by Anderson and Fekete (2001) in which the search space $Q$ is taken to be the planar lattice $Z^{2}$ consisting of points in the plane with integer coordinates, and in each time period they move to (horizontally or vertically) adjacent nodes. The players do not know the initial location of the other, nor do they have a common labeling of the nodes (so for example cannot agree to meet at a specified node). They do not even have a common labeling of the compass directions, so cannot for example agree that in moves 1 to 3 Player I will go North and II will go South. One further question is whether the players have a common notion of clockwise. We consider both answers to this question, calling the version of the rendezvous problem with a common notion of clockwise $\Gamma^{C}$ and the version without it simply $\Gamma$. In each version the rendezvous value $R$ is the minimum expected meeting time, and strategy pairs which achieve this value are called optimal. Clearly $R\left(\Gamma^{C}\right) \leq R(\Gamma)$, since the players have more information in the former case, and we show that both cases (strict inequality or not) can occur. For some distributions of the initial placements, there exist strategy pairs which are uniformly optimal, in the sense that they simultaneously, for all $t$, maximize the probability of meeting by time $t$. Such strategies are very robust. The section organization and main results are as follows.

Section 2 gives a formal definition of the planar rendezvous problems $\Gamma^{C}$ and $\Gamma$ (with and without a common notion of clockwise). A number of necessary conditions for strategies to be optimal or uniformly optimal are proved.

Section 3 analyzes the diagonal initial placement $D$, where the two players are placed at opposite corners of a unit square. In this setting, Anderson and Fekete proved that a modification of the Wait For Mommy strategy is optimal in $\Gamma_{D}^{C}$ (where the $D$ denotes diagonal start). Our main result (Theorem 22 and Corollary 23) is a determination of the full set of optimal strategies for $\Gamma_{D}^{C}$ and $\Gamma_{D}$, and a demonstration that they are all uniformly optimal. In this setting, having a common notion of clockwise does not help the players, as $R\left(\Gamma_{D}^{C}\right)=R\left(\Gamma_{D}\right)$ (rendezvous value is the same in either case).

Section 4 analyzes results obtained in Alpern and Baston (2004a) for parallel initial placement $P$, where the initial vector between the players is of length two and parallel to one of the axes. In this case having a common notion of clockwise does indeed help the players, as $R\left(\Gamma_{P}^{C}\right)<R\left(\Gamma_{P}\right)$. There are no uniformly optimal strategies in either version of the problem. So in both respects, the parallel start version differs from the diagonal start version.

Background on the rendezvous search problem can be found in the survey 
article Alpern (2002a) and in the monograph Alpern and Gal (2003). Of particular relevance to planar rendezvous is the simulation work of Thomas and Hulme (1997). We wish to thank two anonymous referees for useful suggestions which have been incorporated into the current version.

\section{Rendezvous in the Plane: strategies and agents}

We follow the route of Anderson and Fekete by taking the search space $Q$ to be the integer lattice (network) with nodes $z=\left(z_{1}, z_{2}\right) \in Z^{2}$ and nodes are adjacent if they have one coordinate identical and the remaining coordinate differs by 1 . This is just the familiar lattice of graph paper. The distance $d$ between two nodes is defined as the sum of the edges in a shortest connecting path, or equivalently $d\left(\left(z_{1}, z_{2}\right),\left(w_{1}, w_{2}\right)\right)=\left|z_{1}-w_{1}\right|+\left|z_{2}-w_{2}\right|$. At time $t=0$ Nature places the two players on even nodes with the vector $v$ from I to II drawn from a given distribution. (A node $z \in Z^{2}$ is called even if the sum of its coordinates is even; otherwise it is called odd.) In every time period each player must move to an adjacent node or stay still, although we show in Theorem 10 staying still is never optimal. This 'even distance' initial placement (originating in the interval network of Howard (1999)) ensures that the two optimizing players will always have the same parity, and cannot pass each other on an edge without meeting at a node. The players both wish to minimize the expected number of periods required for them to be at the same node.

We analyze the progress of the game in terms of Player I's coordinate system (and sense of clockwise). In this perspective, the initial random placement is achieved by Nature placing I at the origin facing North $(\mathcal{N})$ and placing II at the even node $v_{i}, i=1, \ldots, K$, with probability $p_{i}$, facing equiprobably in either of the four possible directions. (Player II calls this direction $\mathcal{N}$ in any case.) We will consider mainly two particular initial distributions which are both invariant with respect to the group of rotations by $j \pi / 2, j=0,1,2,3$.

Definition 1 The 'parallel' game $\Gamma_{P}$ begins with the initial distribution in which the initial displacement vector between the players has length 2 and is parallel to one of the coordinate axes. Equivalently, $I$ is initially placed at the origin $(0,0)$ and II is initially placed equiprobably at one of the four nodes $v_{1}=(0,2)$, $v_{2}=(2,0), v_{3}=(0,-2), v_{4}=(-2,0)$.

Definition 2 The 'diagonal' game $\Gamma_{D}$ introduced by Anderson and Fekete (2001) begins with the initial distribution in which the players are placed at diagonal corners of a square. Equivalently, I is initially placed at the origin $(0,0)$ and II is placed equiprobably at one of the four nodes $v_{1}=(1,1), v_{2}=(1,-1), v_{3}=$ $(-1,-1), v_{4}=(-1,1)$.

When the game begins, the players have no common notion of locations or directions in the plane. As observers, we adopt I's coordinate system. Now we must distinguish two cases. In the Common Clockwise (CC) case, we assume that the players have a common notion of clockwise (or equivalently, of 'up'). In 
this case a player's orientation is completely determined by his choice of North, and hence there are 4 orientations. In the No Common Clockwise (NCC) case, we don't make this assumption, and must say whether the players have the same notion of clockwise (4 cases) or they don't (another 4 cases). Thus the NCC case has 8 orientations. We denote the $\mathrm{CC}$ game as $\Gamma^{C}$, the $\mathrm{NCC}$ case as simply $\Gamma$. Observe that the $\mathrm{CC}$ rendezvous game $\Gamma^{C}$ has $4 K$ initial configurations (16 for the Diagonal and Parallel games), while the NCC game $\Gamma$ has $8 K$ initial configurations (32 in our examples).

The orientations of player II can be seen as transformations (or rigid motions, or symmetries) of the 'standard orientation' of Player I. In the CC game the four orientations correspond to the four orientation preserving symmetries (preserving the origin) of the planar lattice $Z^{2}$ :

$$
\mathcal{R}_{j}=\text { clockwise rotation by angle } j \pi / 2, j=0,1,2,3 \text {. }
$$

The four $\mathcal{R}_{j}$ correspond to the four possible choices of a North direction by Player II, and the set of these four rotations describes the information symmetry group in the sense of Alpern (1995). Let $v_{i}, i=1, \ldots, K$ be the initial displacements $\left(K=4\right.$ for the parallel or diagonal games $\left.\Gamma_{P}, \Gamma_{D}\right)$. Then the $4 K$ initial configurations for $\Gamma^{C}$ are determined by the $4 K$ values of $i, j$.

We can now define a strategy and show how a pair of strategies determines the meeting times of the two players, one for each initial configuration. (We begin by allowing the null move $(0,0)$, but we will remove this possibility later.)

Definition 3 A strategy for a player (in either game $\Gamma^{C}$ or $\Gamma$ ) is a sequence of directions $D_{i} \in\{(0,0), N=(1,0), E=(1,0), S=(0,-1), W=(-1,0)\}, i=$ $1,2, \ldots$. A player pursuing this strategy moves successively one unit in his direction $D_{0}, D_{1}, \ldots$, according to his initial orientation. Equivalently, it can be seen as his net displacement $f(t)$ at time $t$ from his initial location, given by $f(0)=(0,0)$ and for $t \geq 1$,

$$
f(t)=\sum_{k=1}^{t} D_{k} .
$$

So for example the strategy beginning $N, E, E$, corresponds to a net displacement function $f$ with

$$
[f(0), f(1), f(2), f(3)]=[(0,0),(0,1),(1,1),(2,1)] .
$$

We shall deal with strategy pairs $(f, g)$ where Player I adopts $f$ and II adopts $g$. Sometimes we will use the symmetric notation $\left(f_{1}, f_{2}\right)$. In this setting, the location of Player I at time $t$ is simply $f(t)$, while the location of II (in I's coordinate system) depends on the initial configuration, as described below.

We begin by considering the common clockwise (CC) game $\Gamma^{C}$. If the initial configuration gives Player II initial location $v_{i}$ and orientation $\mathcal{R}_{j}$ then the location of Player II at time $t$ under strategy $g$ is given by

$$
g_{i, j}(t)=v_{i}+\mathcal{R}_{j}(g(t)) .
$$


Definition 4 The $4 K$ (in our examples, 16) paths $g_{i, j}$ are called the agents of Player II. We call $g_{i j}$ the agent starting at $v_{i}$ in direction $j$, where directions $j=0,1,2,3$, are $\mathcal{N}, \mathcal{E}, \mathcal{S}, \mathcal{W}$ (that is, $\mathcal{R}_{j}(N=(0,1)$ ). Each agent from $v_{i}$ is the actual path of player II with probability $p_{i} / 4$. (Note that we use the typeface $\mathcal{E}$ to indicate an agent initially facing East, and the typeface $E$ to indicate a move East.)

The time taken for agent $g_{i, j}$ to be met by Player I is called its meeting time, and denoted

$$
\omega_{i, j}(f, g)=\min \left\{t: f(t)=g_{i, j}(t)\right\},
$$

and the time required to meet all the agents is called $M(f, g)$, where

$$
M(f, g)=\max _{i, j} \omega_{i, j}(f, g) .
$$

Figure 1 shows the 16 initial configurations in $\Gamma_{P}^{C}$, and Figure 2 shows the strategy pair starting with $W S$ for I and $N E$ for II, with the paths of I (thick line) and of all 16 agents of II for $t=0,1,2$. Observe that at time $t=1$ Player I meets the agent $g_{4,1}$ of II starting at $v_{4}=(-2,0)$ and facing $\mathcal{E}$ (whose North is the direction that $I$ calls East $\left.\left(\mathcal{R}_{1}\right)\right)$, hence $\omega_{4,1}=1$. Similarly at time $t=2$ he meets the agent $g_{3,3}$ who started at $v_{3}=(0,-2)$ and whose North is what I calls West $\left(\mathcal{R}_{3}\right)$, so that $\omega_{3,3}=2$. All other meeting times are greater than 2 .

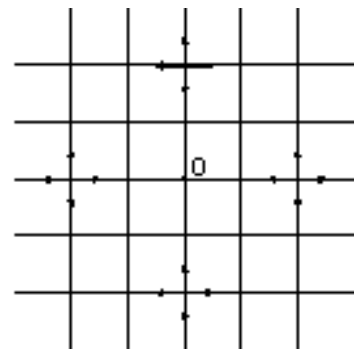

Figure 1: 16 agents in $\Gamma_{P}^{C}$

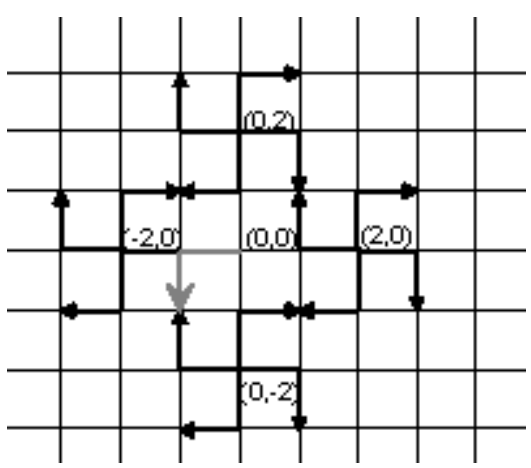

Figure 2: Strategy $(W, S),(N, E)$ 
Note that in Figure 2 the agents of II starting at the same node are at four distinct locations at each time $t=1,2$. This will be true as long as they are not at their starting point (that is, $g \neq 0$ ), since the group of four rotations gives four distinct locations when applied to points other than the origin. Thus we have the following useful fact.

Remark 5 In $\Gamma^{C}$, Player I can never simultaneously meet more than one agent of Player II from the same starting point unless he is at that starting point. That $i s, \omega_{i j}=\omega_{i j^{*}}=t, j \neq j^{*}$ implies $f(t)=g_{i j}(t)=g_{i j^{*}}(t)=v_{i}$.

This motivates the following definition.

Definition 6 A meeting between Player I and an agent $g_{i, j}$ of Player II which takes place at node $z$ is called a Starting Point Meeting (SPM) if $z$ is the starting point of I (the origin) or the starting point $v_{i}$ of that agent of II. In the former case it is called type I, in the latter, type II.

Given a strategy pair $(f, g)$, the average value of the meeting times $\omega$ is called $T^{C}(f, g)$. Thus

$$
\begin{aligned}
T^{C}(f, g) & =\frac{1}{4} \sum_{i, j} p_{i} \omega_{i, j}(f, g), \text { for } \Gamma^{C}, \text { or simply } \\
& =\frac{1}{16} \sum_{i, j} \omega_{i, j}(f, g) \text { for diagonal or parallel start. }
\end{aligned}
$$

The rendezvous value $R$ for the CC game $\Gamma^{C}$ is the least expected time,

$$
R\left(\Gamma^{C}\right)=\min _{f, g} T^{C}(f, g),
$$

and any pair $f, g$ achieving the minimum is called optimal for $\Gamma^{C}$.

We now consider the NCC game $\Gamma$. This game now has $8 \mathrm{~K}$ agents for II, rather than $4 \mathrm{~K}$. To each agent $g_{i, j}$ who has the same clockwise orientation as I (since rotations preserve orientation in this sense) there is an associated agent $g_{i, j}^{\prime}$ with the same starting point and the same notion of $N$, but who has the opposite notion of clockwise orientation (or equivalently, $E$ and $W$ reversed). To every strategy $g$ defined by a move sequence we define an associated strategy $g^{\prime}$ which is the same as $g$ but has $E$ and $W$ moves interchanged. For example, if

$$
g=[N, E, E, N, S, W, \ldots], \text { then } g^{\prime}=[N, W, W, N, S, E, \ldots] .
$$

If II is following strategy $g$, he has $4 K$ agents $g_{i, j}$ and $4 K$ agents $g_{i, j}^{\prime}$, so $8 \mathrm{~K}$ agents in all. (So if there are $K=4$ starting points, as in the two examples we consider, there are 32 agents in $\Gamma$.) We call the associated meeting times $\omega_{i, j}$ and $\omega_{i, j}^{\prime}$. The expected meeting time in $\Gamma$ for a strategy pair $(f, g)$ is given by

$$
\begin{aligned}
T(f, g) & =\frac{1}{8 K}\left(\sum_{i, j} \omega_{i, j}(f, g)+\sum_{i, j} \omega_{i, j}^{\prime}(f, g)\right) \\
& =\frac{1}{2}\left(T^{C}(f, g)+T^{C}\left(f, g^{\prime}\right)\right) .
\end{aligned}
$$


In a similar fashion to the $\mathrm{CC}$ game, we define the $\mathrm{NCC}$ rendezvous value

$$
R(\Gamma)=\min _{f, g} T(f, g), \text { and any pair }
$$

$f, g$ achieving the minimum is called optimal for $\Gamma$.

It follows from (6) and the fact that the minimum of $T^{C}(f, g)$ and $T^{C}\left(f, g^{\prime}\right)$ is less than or equal to their average that

Proposition $7 R\left(\Gamma^{C}\right) \leq R(\Gamma)$, with equality if and only if $\Gamma^{C}$ has an optimal strategy $(f, g)$ for which $\left(f, g^{\prime}\right)$ is also optimal. In the case of equality $R\left(\Gamma^{C}\right)=$ $R(\Gamma)$, a strategy $(f, g)$ is optimal in $\Gamma$ if and only if both $(f, g)$ and $\left(f, g^{\prime}\right)$ are optimal in $\Gamma^{C}$.

Equality holds for the diagonal start game $\Gamma_{D}$ (Section 3) while strict inequality holds for the parallel start game $\Gamma_{P}$ (Section 4).

The 'Wait for Mommy' (WFM) strategy pair, where II stays still while I takes a minimal path reaching all the starting points $v_{i}$ of $I I$, has all finite meeting times $\omega$ (in CC or NCC) and consequently a finite expected meeting time $T$. Hence the rendezvous times $R$ and $R^{C}$ are both finite. So we restrict our search for optimal strategies to exhaustive strategy pairs, those with finite maximal search times. Exhaustive strategy pairs can be given by a finite sequence of directional moves. A stronger notion of optimality is the following.

Definition 8 A strategy pair (for either $\Gamma$ or $\Gamma^{C}$ ) is called uniformly optimal if for all $t$ it maximizes the probability that the players have met by time $t$. (Note that if there is a uniformly optimal strategy, then all optimal strategies must be uniformly optimal.)

A uniformly optimal strategy maximizes the expected utility of the meeting time $\omega$ as long as the utility function is non-increasing in $\omega$ (earlier meetings are preferred to later ones); an optimal strategy is only required to accomplish this for the particular utility function $-\omega$.

Lemma 9 If $(f, g)$ and $\left(f, g^{\prime}\right)$ are uniformly optimal for $\Gamma^{C}$, then they are uniformly optimal for $\Gamma$.

Proof. Suppose on the contrary that for some strategy $(\breve{f}, \breve{g})$ in $\Gamma$, and some time $t$, the probability that the players have met, $\left(\breve{p}+\breve{p}^{\prime}\right) / 2$, exceeds the corresponding probability $p$ for $(f, g)$. Then either $\breve{p}$ or $\breve{p}^{\prime}$ exceeds $p$, and so either $(\breve{f}, \breve{g})$ or $\left(\breve{f}, \breve{g}^{\prime}\right)$ contradicts the uniform optimality of $(f, g)$ in $\Gamma^{C}$.

We have been assuming for the sake of generality that players may stay still (the null choice of move $(0,0)$ in certain periods). However, restricting moves to the four compass directions reduces the size of the strategy set, so we henceforth will not allow players to stay still. This does not remove any otherwise optimal strategies, as shown in the following. 
Theorem 10 Let $\left(f_{1}, f_{2}\right)$ be an optimal strategy pair for the rendezvous problem $\left(\Gamma^{C}\right.$ or $\left.\Gamma\right)$. Define $\omega^{0}=0$ and let $\omega^{1}<\omega^{2}<\cdots<\omega^{K}$ denote the associated set of meeting times with the $(4 K$ or $8 K)$ agents, listed in increasing order. Let $d$ denote the graph distance on the lattice $Z^{2}$. Then

$$
d\left(f_{i}\left(\omega^{m-1}\right), f_{i}\left(\omega^{m}\right)\right)=\omega^{m}-\omega^{m-1}, \text { for } i=1,2 \text { and } m=1, \ldots, K .
$$

In other words, both players move in time-minimizing paths between consecutive meeting points. In particular, neither player ever stays still, and consequently both players are at even (odd) nodes at all even (odd) times.

Proof. Suppose the condition (9) fails for some minimum number $m$, and let $g_{\alpha}$ be an agent that $I$ meets at time $M=\omega^{m}$. By the minimality of $m$, the players are at nodes of the same parity (and an even distance apart) at time $\omega^{m-1}$. Let $z$ denote the meeting node (I's location) at time $M$. We are assuming that at least one of the players can get to $z$ prior to time $M$ (given his location at the earlier time $\omega^{m-1}$ ). If both players can get to $z$ before $M$, let them do so and stay there through time $M$, when they resume there original paths. This strategy modification brings forward the meeting time with agent $g_{\alpha}$ without postponing any other meeting times, contradicting the assumed optimality of the strategy pair. Otherwise only one player (which we may assume is Player I, by renaming) can get to $z$ prior to $M$. So modify I's strategy so that he gets to $z$ at the earliest possible time $M-n$. At time $M-n$, agent $g_{\alpha}$ will be at some node $b$, at distance $n$ from node $z$, and since both players have still been moving on every step up to time $M-n$, their distance $n$ at time $M-n$ must still be even, $n=2 j$. Next let Player I move to meet $g_{\alpha}$ at time $M-j$, and then follow $g_{\alpha}$ back to $z$ at the original time $M$. Again, this moves the meeting with $g_{\alpha}$ forward by $j>0$ steps, without postponing any other meeting, again contradicting the assumed optimality of the strategy.

Remark 11 This result is similar to Theorem 1 of Alpern (2002b) for labeled graphs (or Theorem 13.2 of Alpern and Gal (2003)). It can also be considered a generalization of the similar result for the line, Theorem 16.10 of Alpern and Gal (2003). See also Gall (1999) for non-optimality of staying still. 


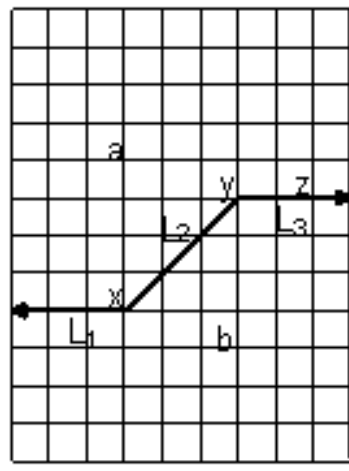

$\left|a_{1}-b_{1}\right|<\left|a_{2}-b_{2}\right|$

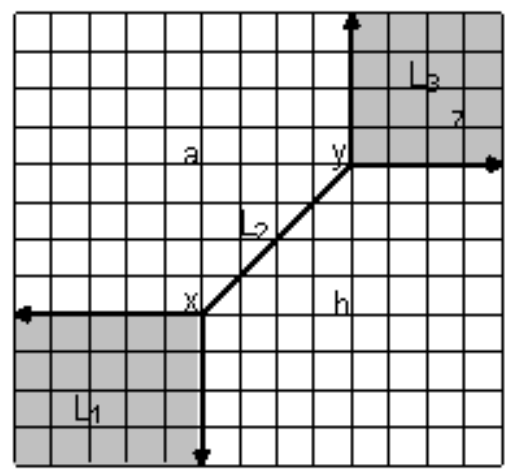

$\left|a_{1}-b_{1}\right|=\left|a_{2}-b_{2}\right|$

Figure 3: Midpoint, equidistant sets for $a, b$.

Figure 3 illustrates the previous theorem. At time $\omega^{m-1}$, I is at node $a$ and agent $\alpha$ of II is at node $b$, where $d(a, b)=2 l$ (in both figures, $l=4$ ). In the time interval $\left[\omega^{m-1}, \omega^{m}\right]$ of length $k=\omega^{m}-\omega^{m-1} \geq l$ (in the figures, $k=6,8$ ), both I and agent $\alpha$ follow geodesics to a common node $z$ with $d(a, z)=d(b, z)=k$, where they meet at time $\omega^{m-1}+k$. The node $z$ belongs to the equidistant set

$$
E(a, b)=\{z: d(a, z)=d(b, z)\}=L_{1} \cup L_{2} \cup L_{3} .
$$

We now further claim that if the strategy pair is optimal, $z$ must in fact belong to the midpoint set

$$
\operatorname{Mid}(a, b)=L_{2}=\left\{z \in Z^{2}: d(a, z)=d(b, z)=\frac{d(a, b)}{2}=l\right\}
$$

so that $k$ is equal to $l$. If not, then $z$ belongs to $L_{1}$ or $L_{3}$. Assume without loss of generality that $z$ belongs to $L_{3}$. Suppose we modify the paths taken by I and agent $\alpha$ of II so that they each follow a geodesic to $z$ that goes through the node $y$. Then they both get to $z$ at the original time $\left(\omega^{m-1}+k\right)$ but they meet at the earlier time $\left(\omega^{m-1}+l\right)$. The resulting modified strategy pair has the meeting with agent $\alpha$ brought forward by $k-l$ periods, without postponing any other meeting. So the original strategy cannot have been optimal.

Corollary 12 Let $\left(f_{1}, f_{2}\right)$ be an optimal strategy pair for the rendezvous problem $\left(\Gamma^{C}\right.$ or $\left.\Gamma\right)$. Define $\omega^{0}=0$ and let $\omega^{1}<\omega^{2}<\cdots<\omega^{M}$ denote the set of meeting times for $\left(f_{1}, f_{2}\right)$, listed in increasing order. Suppose that Player I meets agent $\alpha$ of II at time $\omega^{m}$. Then their meeting point $z=f_{1}\left(\omega^{m}\right)=g_{\alpha}\left(\omega^{m}\right)$ is a midpoint of their locations at time $\omega^{m-1}$ and occurs at the earliest possible time. That is,

$d\left(z, f_{1}\left(\omega^{m-1}\right)\right)=d\left(z, g_{\alpha}\left(\omega^{m-1}\right)\right)=\frac{1}{2} d\left(f_{1}\left(\omega^{m-1}\right), g_{\alpha}\left(\omega^{m-1}\right)\right)=\omega^{m}-\omega^{m-1}$. 
Note in particular that this result applies to every agent that I meets at time $\omega^{m}$, so if there are several, the meeting must take place at a common midpoint of the locations of these agents and I at $\omega^{m-1}$. This result says that the time interval $\omega^{m}-\omega^{m-1}$ is minimized, given the situation at time $\omega^{m-1}$ and the choice of $\alpha$ as the next agent to be met. However the possibility exists that $\omega^{m}-\omega^{m-1}$ could be made smaller if a different agent $\beta$ (closer to I than $\alpha$ is at time $\omega^{m-1}$ ) would be the next to be met. However this is not possible if $\left(f_{1}, f_{2}\right)$ is uniformly optimal, since choosing to meet agent $\beta$ next at time $\omega^{*}$ with $\omega^{m-1}<\omega^{*}<\omega^{m}$ would yield a strategy pair with a higher probability than the original strategy of meeting by time $\omega^{*}$, and so $\left(f_{1}, f_{2}\right)$ could not have been uniformly optimal. Thus we have established the following 'greedy' property of uniformly optimal strategies.

Theorem 13 Let $\left(f_{1}, f_{2}\right)$ be a uniformly optimal strategy pair for the rendezvous problem $\left(\Gamma^{C}\right.$ or $\left.\Gamma\right)$. Define $\omega^{0}=0$ and let $\omega^{1}<\omega^{2}<\cdots<\omega^{M}$ denote the set of meeting times for $\left(f_{1}, f_{2}\right)$, listed in increasing order. Then at time $\omega^{m}$, I meets one of the agents of II who was at minimum distance to him at time $\omega^{m-1}$, and $\omega^{m}-\omega^{m-1}$ is half of that distance.

\section{Planar Rendezvous with Diagonal Start}

In this section we analyze the diagonal start (Figure 4) game $\Gamma_{D}$ introduced by Anderson and Fekete, and extend some of their pioneering results.

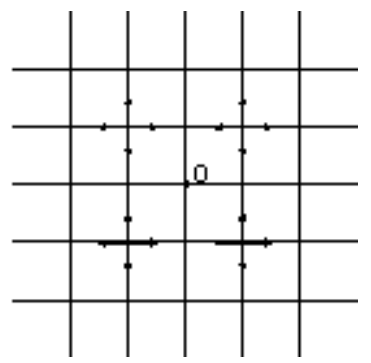

Figure 4: Start in $\Gamma_{D}^{C}$

Anderson and Fekete (2001) analyzed a strategy pair which we call the AF strategy, given by $\bar{f}=[N, E, S, S, W, W, N, N], \bar{g}=[N, S, N, S, N, S, N, S]$. Note that since II's strategy $\bar{g}$ has no $E$ or $W$ moves, it satisfies the invariance equation $\bar{g}^{\prime}=\bar{g}$, and by (6) has the same expected meeting time with or without the assumption of common clockwise, $T(\bar{f}, \bar{g})=T^{C}(\bar{f}, \bar{g})$. The following table indicates the meeting times $\omega_{i, j}$ corresponding to $(\bar{f}, \bar{g})$ depending on the initial 
direction which II calls North and the initial location of Player II.

\begin{tabular}{|c||c|c|c|c|}
\hline Player II & & initial & direction & \\
\hline Starting Point & $\mathcal{N}$ & $\mathcal{E}$ & $\mathcal{S}$ & $\mathcal{W}$ \\
\hline \hline$(1,1)$ & 2 & 2 & 2 & 1 \\
\hline$(1,-1)$ & 3 & 4 & 4 & 4 \\
\hline$(-1,-1)$ & 6 & 5 & 6 & 6 \\
\hline$(-1,1)$ & 8 & 1 & 7 & 8 \\
\hline
\end{tabular}

For each time $t=1, \ldots, 8$, the number of entries of the $4 \times 4$ matrix of meeting times which are equal to $t$ is denoted by $\bar{x}_{t}$ and the number which are less than or equal to $t$ is denoted by $\bar{y}_{t}$. Thus in the A-F strategy Player I meets $\bar{x}_{t}$ of the 16 Player II agents at time $t$ and $\bar{y}_{t}$ of these agents by time $t$. For a general strategy we will let $x_{t}$ and $y_{t}$ denote these numbers. For the A-F strategy we have

$\begin{array}{ccccccccc}t & 1 & 2 & 3 & 4 & 5 & 6 & 7 & 8 \\ \bar{x}_{t} & 2 & 3 & 1 & 3 & 1 & 3 & 1 & 2 \\ \bar{y}_{t} & 2 & 5 & 6 & 9 & 10 & 13 & 14 & 16\end{array}$

The expected meeting time for the A-F strategy $(\bar{f}, \bar{g})$ is

$$
T^{C}(\bar{f}, \bar{g})=\frac{1 \cdot 2+2 \cdot 3+3 \cdot 1+4 \cdot 3+5 \cdot 1+6 \cdot 3+7 \cdot 1+8 \cdot 2}{16}=\frac{69}{16} .
$$

Anderson and Fekete established that their (A-F) strategy is optimal in $\Gamma^{C}$ - no strategy gives a lower expected meeting time. We will prove a stronger result, namely that it is uniformly optimal in $\Gamma^{C}$ and in $\Gamma$. To do this we will need to use part of their original proof, which we give below, with an additional useful inequality in the statement which is due to an anonymous referee.

Lemma 14 For any optimal strategy pair $(f, g)$ in $\Gamma_{D}^{C}$, the number of meetings $x_{i}$ at time $i$ satisfies $x_{1}=2$, and $x_{i} \leq 3$, for any $i$. Furthermore, if $x_{i}=3$, then $i$ is even, Player I is at his own or one of II's starting locations at time $i$ (a Starting Point Meeting of type I or II, respectively) and $x_{i+1} \leq 1$. Consequently for all even $i$ we have $x_{i}+x_{i+1} \leq 4$.

Proof. Since we may assume without loss of generality that both strategies begin with $N$, we simply observe that at time 1 , Player I will meet the $\mathcal{E}$ agent starting at $(-1,1)$ and the $\mathcal{W}$ agent starting at $(1,1)$ and no other agents, so $x_{1}=2$. Suppose that $x_{i} \geq 3$, which means that at time $i$ Player I meets at least three agents of II at some location $A$. We first show that at time $i$ one of the players must be back at his start. Suppose not. Then by Remark 5, all the agents that I meets at time $i$ must come from different starting points. Since all Player II agents are equally distant from their respective starting positions, the node $A$ must be equally distant (in the Manhattan or graph distance) from at least three of the starting points of II. The only such location is the origin, that is, Player I's initial location. So the meeting must be at a starting point, and hence by Theorem 10, $i$ must be even. By symmetry of the players, we will 
assume that $A$ is one of the starting points of II. At time $i-1$ both Player I and one of the agents of II who started at $A$ must be at the same location. Hence $x_{i} \leq 3$ as claimed. Since all agents of II must be at their starting points at time $i$, and $\mathrm{I}$ is at one of these, he can meet at most one agent of II at time $i+1$.

Theorem 15 The $A-F$ strategy $(\bar{f}, \bar{g})$ is uniformly optimal in the 'common clockwise' diagonal start game $\Gamma_{D}^{C}$. Consequently any optimal strategy must have $x=\bar{x}=(2,3,1,3,1,3,1,2), y=\bar{y}=(2,5,6,9,10,13,14,16)$, and hence by the previous Lemma there must be starting point meetings at time 2, 4, and 6 .

Proof. We have to show that the cumulative meeting probability sequences $y=\left(y_{1}, \ldots, y_{8}\right)$ corresponding to any strategy pair is bounded above by the one corresponding to the A-F strategy, namely $y_{t} \leq \bar{y}_{t}$. This is obvious for $t=1$ (where equality holds by Lemma 14) and $t=8$ (where $\bar{y}_{t}$ is maximal). For $t=2 j+1$, and $t=2 j, j=1,2,3$, we have from Lemma 14 that

$$
\begin{aligned}
y_{2 j+1} & =x_{1}+\sum_{i=1}^{j}\left(x_{2 i}+x_{2 i+1}\right) \leq 2+4 j=\bar{y}_{2 j+1}, \text { and } \\
y_{2 j} & =x_{1}+\sum_{i=1}^{j-1}\left(x_{2 i}+x_{2 i+1}\right)+x_{2 j} \leq 2+4(j-1)+3=\bar{y}_{2 j} .
\end{aligned}
$$

Corollary 16 The $A-F$ strategy $(\bar{f}, \bar{g})$ is uniformly optimal in the diagonal start 'no common clockwise' game $\Gamma_{D}$. Consequently $R\left(\Gamma_{D}\right)=R\left(\Gamma_{D}^{C}\right)=69 / 16$.

Proof. Since $\bar{g}^{\prime}=\bar{g}$ for the A-F strategy, the previous result says that both $(\bar{f}, \bar{g})$ and $\left(\bar{f}, \bar{g}^{\prime}\right)$ are uniformly optimal in $\Gamma_{D}^{C}$, so the result follows from Lemma 9 .

We now determine the full set of optimal strategies $(f, g)$ for $\Gamma_{D}^{C}$. Suppose that $(f, g)$ is such a strategy pair. According to Theorem $15,(f, g)$ must have the meeting time sequence $(2,3,1,3,1,3,1,2)$ and there must be SPM's at times 2,4 , and 6 . There must also be an SPM at time 8 (Theorem 20). We can classify optimal strategies according to where these meetings take place (at the starting point of I or II) For example, the A-F strategy has 'meeting type' [II,II,II,II]. We will assume without loss of generality that II is at home at $t=2$ so the meeting type will always begin with a II. Optimal strategy pairs for $\Gamma_{D}^{C}$ remain optimal if either strategy element is rotated, so we may assume they both start with an $N$. Since I visits a starting point of II at time 2 , he must make a turn at time 1 , so we may assume his strategy starts with $(N, E, \ldots)$. Similarly, since II is back at his start at time 2 , we may assume he starts with $(N, S, \ldots)$. Strategy pairs starting in this way are called 'standard', or in 'standard form'. Every optimal strategy can be obtained from a standard one by rotating $f$ and $g$. Our classification (Theorem 20) of meeting types requires three lemmas.

Lemma 17 If a standard optimal strategy for $\Gamma_{D}^{C}$ has a Starting Point Meeting (SPM) of type I at time 4, then it has an SPM of type II at time 6. 
Proof. At any (odd) time there can be at most two agents from the same starting point adjacent to $(0,0)$. Now all the agents of $(1,1)$ have been met by time 2. Thus if there is an SPM of type I at time 4, there can be at most three unmet agents adjacent to $(0,0)$ at time 5 . Hence, if player I is also at $(0,0)$ at time 6 (type I SPM), he can meet a total of at most 3 agents on moves 5 and 6 which contradicts (Theorem 15) the required meeting pattern of $(2,3,1,3,1,3,1,2)$, which has $x_{5}+x_{6}=1+3=4$. Since an SPM of some type at time 6 is guaranteed by Lemma 14 ( since $x_{6}=3$ ), it must be of type II.

Lemma 18 If a standard optimal strategy for $\Gamma_{D}^{C}$ has a Starting Point Meeting (SPM) of type II at time 6 , then it has an SPM at time 8.

Proof. Any optimal strategy must have $x_{8}=2$, by Theorem 15. If the two agents met at time 8 have the same starting point then, by Remark 5 , they can only be met at that starting point, and we are done. If they have different starting points $G$ and $H$, these must be distinct from a third starting point $J$ where the SPM occurs at time 6 . Hence the meeting at time 8 must occur at a node $z$ whose distance from all three nodes $G, H$, and $J$ is no more than 2. Hence $z$ is the origin, again with an SPM at time 8.

Lemma 19 If a standard optimal strategy for $\Gamma_{D}^{C}$ has a Starting Point Meeting (SPM) of type II at time 4, then it has SPM's of type II at times 6 and 8 as well. That is, if the meeting type begins [II, II, ...], then it is [II, II, II, II]. In this case Player I's strategy is $(N, E, S, S, W, W, N, N)$.

Proof. Since there are SPM's of type II at times 2 (by definition of standard) and 4 (by hypothesis), it follows that at any time $t>4$ at most two agents (one each from the two starting points not visited at times 2,4 ) can be met at the origin. Consequently, since $x_{6}=3$, the SPM at time 6 (guaranteed by Lemma 15) must be of type II (not at origin). It now follows from Lemma 18 that there is an SPM at time 8. However the type II SPM's at times 2, 4, and 6, imply that if this SPM is of type I, then at most one agent can be met at time 8 (from the one starting point not visited). However $x_{8}=2$ for any optimal strategy (Theorem 15). Hence the SPM at time 8 must be of type II.

Theorem 20 Every standard optimal strategy for $\Gamma_{D}^{C}$ has $S P M$ 's at $t=2,4,6,8$, and the meeting types [II, I, II, II], [II, I, II, I], or [II, II, II, II].

Proof. The meeting type must begin [II,...] by definition of 'standard'. If the second SPM is of type I then Lemma 17 implies the next one must be of type II, so Lemma 18 gives the two allowed cases [II, I, II, II] and [II, I, II, I]. If the second SPM is of type II, then by Lemma 19 the meeting type is [II, II, II, II].

Figure 5 gives the full list of standard optimal strategies for $\Gamma_{D}^{C}$, grouped by meeting type. 

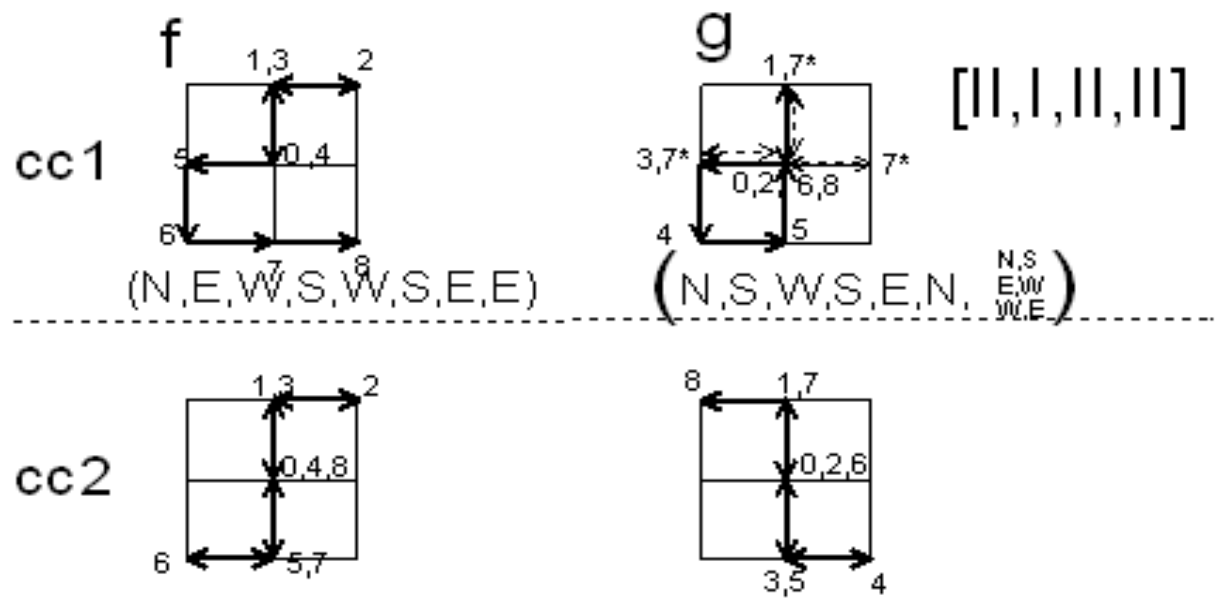

(N,E,W,S,S,W,E,N)

$(N, S, S, E, W, N, N, W)$
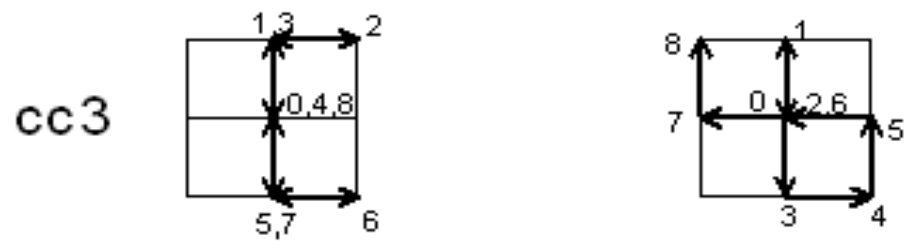

(N,E,W,S,S,E,W,N)

(N,S,S,E,N,W,W,N)
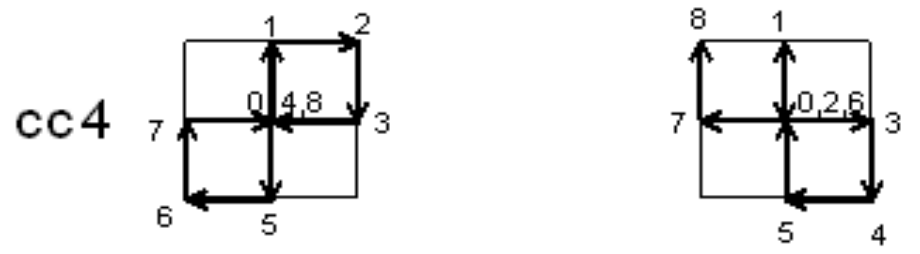

$[I I, I, I I, I]$

(N,E,S,W,S,W,N,E)

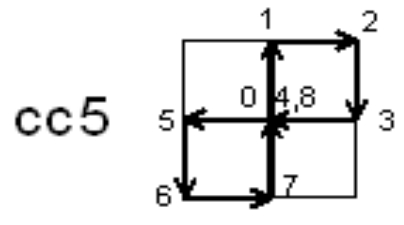

(N,S,E,S,W,N,W,N)

(N,E,S,W, W, E,N)
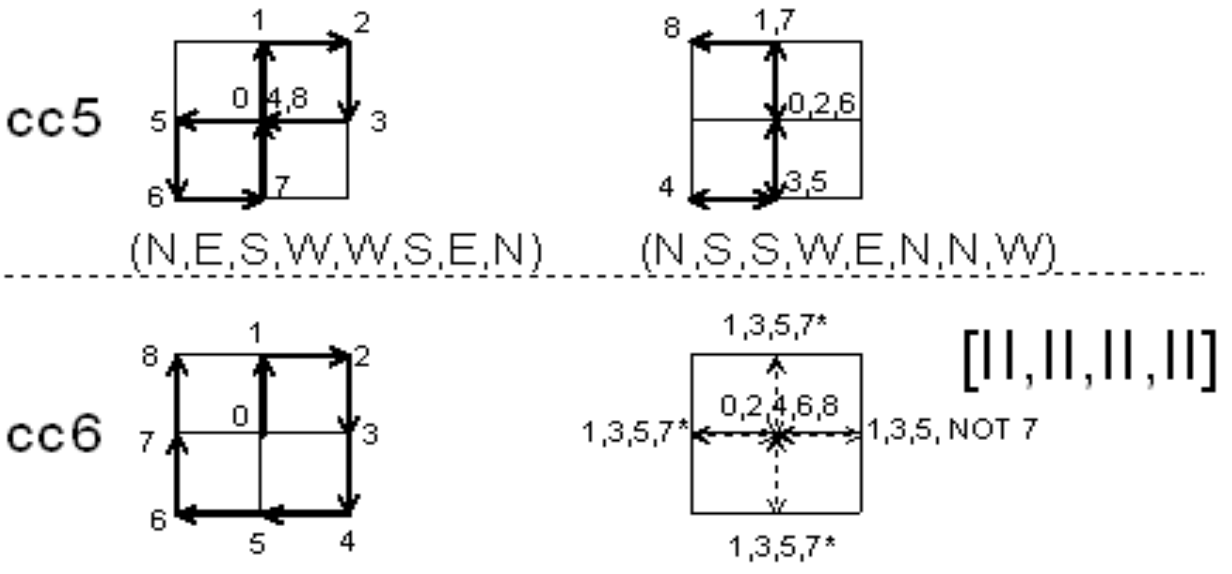

(N,E,S, S, W, W,N, 13 )

$$
(N, S, D 3,-D 3, D 5,-D 5, \underset{W, N}{S, N})
$$

Figure 5: Full set of standard optimal strategies fo $\Gamma_{D}^{C}$. 
There are six families of strategies, called $c c 1$ to $c c 6$. The middle group, with meeting type [II,I,II,I], has exactly four strategy pairs. The solid lines indicate the player's path relative to his coordinate system (with his origin at the center and his North drawn 'up'). The numbers indicate the time when the player reaches a given location. Strategy pair $c c 4$ is the A-B strategy drawn in Figure 18.2 of Alpern and Gal (2003) (but written incorrectly there above the figure and with mistakes in the table on p.282.) The top group, [II,I,II,II], has three strategy pairs, all with the same $f$. Player II has three possibilities at moves 7 and 8 , indicated by dashed lines. His possible locations at time 7 are indicated by $\mathrm{a}^{*}$. The bottom group, with meeting type [II,II,II,II] consist of Modified Wait For Mommy strategies, where player I searches all the possible initial locations of II (at times $2 i, i=1, \ldots, 4$ ), while II is back at his start at these times. The only restriction on II's motions $(g)$ is that his move at time 7 cannot be E. The reason for this is that I meets the agent starting at $(-1,1)$ facing $\mathcal{E}$ at time 1 at I's location $(0,1)$, and must meet a different agent who started at $(-1,1)$ at time 7 and I's location $(-1,0)$. This is best seen by referring to the bottom left drawing of Figure 5. In order to insure that the agent who went East (in I's system) from $(-1,1)$ at time 1 (and is no longer alive) is not the same one who goes South (in I's system) at time 7, we must require that the direction for II at time 7 is not a 'right turn' from his direction at time 1 . Since we are taking the latter as $N$, we exclude $E$ (a right turn from $N$ ) at time 7 . It is easily verified that all the strategies $c c 1$ to $c c 6$ have the meeting time profile $(2,3,1,3,1,3,1,2)$ and are therefore optimal. That there are no additional optimal strategies for the common clockwise problem $\Gamma_{D}^{C}$ is established by the following results, with some cases illustrated below.

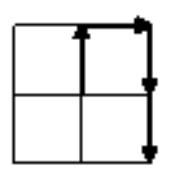

a: NESS

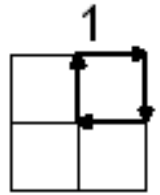

b: NESW

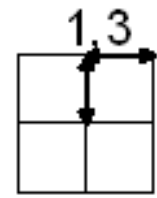

c:NEWS

Figure 6: Situation after four moves of Player I

We begin by showing that the left (' $f$ ') column of Figure 5 contains all the optimal strategies for I, and then we will show that to each of these $f$ 's the only complementary (optimal) $g$ 's are those drawn on its right.

Theorem 21 If $(f, g)$ is a standard optimal strategy for the common clockwise, diagonal start, rendezvous problem $\Gamma_{D}^{C}$, then its first component $f$ is one of the $f$ strategies in $c c 1$ to $c c 6$ defined in Figure 5. 
Proof. By Theorem 20 we need only consider the three meeting types [II, II, II, II], [II, I, II, II], and [II,I,II,I]. If the meeting type is [II, II, II, II], we have already established in Lemma 19 that the Anderson-Fekete strategy (cc6) for I of $(N, E, S, S, W, W, N, N)$ is the only possibility.

Consider the meeting type [II, I, II, II]. In this scenario, I visits three of II's starting nodes. The only way he can meet all four agents of the starting point $v$ not visited, is to meet one at the origin at time 4 , and to meet the three others at distinct (by Remark 5) times at nodes adjacent to $v$. So in particular I's path must be adjacent to the unvisited starting point $v$ at three times $t$. Now I must begin with $(N, E)$ (by definition of standard) and return to the origin at time 4 by continuing either $(N, E, S, W, \ldots)$ or $(N, E, W, S)$. If the former (Figure $6 \mathrm{~b}$ ), his two remaining starting points to visit cannot be $(-1,1)$ and $(1,-1)$ because the distance between them is 4 , the time remaining. Hence he must visit one of those and $(-1,-1)$, and be adjacent to the remaining one on two more occasions. Since this is not possible, he must begin $(N, E, W, S, \ldots)$, as in Figure 6c. Player I has now been adjacent to starting point $(-1,1)$ twice $(t=1,3)$, so he needs to be adjacent one more time and also visit the starting points $(-1,-1)$ and $(1,-1)$ (in some order). The only path with this property is clearly that of cc1, namely $(N, E, W, S, W, S, E, E)$.

We now show that the meeting type [II,I,II,I] restricts $f$ to the four possibilities $c c 2$ to $c c 5$. By the same reasoning as in the previous paragraph, Player I must visit two starting points $(t=2,6)$ and be adjacent to each of the other two on two occasions (and at the origin $t=4,8$ ). Starting with $(N, E)$ (as required by the definition of standard), I can return from $(1,1)$ to $(0,0)$ with either continuation $(N, E, W, S)$ or $(N, E, S, W)$. In the former case (Figure 6c), by time 4 he has already visited the node $(0,1)$ adjacent to starting point $(-1,1)$ twice. If he visits starting point $(-1,1)(t=6)$ and is back at the origin at time 8 , his path will obviously not have the required property. So he must be at $(-1,0)$ for $t=5,7$, and at $t=6$ either at $(-1,-1)(c c 2)$ or $(1,-1)(c c 3)$.

If Player I begins $(N, E, S, W, \ldots)$, as in Figure $6 \mathrm{~b}$, he has already been adjacent to both starting points $(-1,1)$ and $(1,-1)$. So the continuation must pass through $(-1,0),(0,-1)$ (adjacent to the two unvisited starting points), $(-1,-1)$ (the remaining starting point), and return to the origin at time 8 . To do this, he must go around the square with diagonal $(-1,-1)$ and the origin in either in the clockwise $(c c 4)$ or counter-clockwise $(c c 5)$ direction.

Theorem 22 For each of the six Player I strategies $f$ listed in Figure 5 as $c c 1$ to $c c 6$, the standard strategies $g$ listed to its right are the only ones for which $(f, g)$ is optimal. Consequently the strategy pairs in Figure 5 constitute the complete set of (uniformly) optimal standard form strategies for the diagonal start, common clockwise, rendezvous problem $\Gamma_{D}^{C}$.

Proof. We must show that for each of the $f$ strategies (in the left column of Figure 5) which were shown in Theorem 21 to be potentially optimal, the $g$ 's on the right constitute the full set which form an optimal pair. We have already shown this for the Modified Wait For Mommy Strategies (meeting type 
[II,II,II,II] in the paragraph following Figure 5: Player II must be back at his start at all even times $t$, and he cannot move $S$ (opposite to his first move) on move 7 . It remains to demonstrate the Theorem for meeting types [II,I,II,II] and [II,I,II,I] .

Meeting type [II,I,II,II]: For this part of the proof, we maintain our usual perspective of I's coordinate system. So I starts at $(0,0)$ and seeks to meet all 16 agents of II, four each from each starting node $v_{i}$. If Player I is following $f_{1}$ (the $f$ of $c c 1$ ), then he is at locations $a=(0,1), b=(0,0)$, and $c=(-1,0)$ at times $t=3,4,5$, respectively, and must meet the three remaining agents of II starting at $v_{4}=(-1,1)$ (aside from $\mathcal{E}$, who was met at time $t=1$ ) at these times (the only times when $\mathrm{I}$ is adjacent to $v_{4}$.) At time $t=2$, these agents are all at their start $v_{4}$, so at time $t=3$ one of them goes to $a$, another goes to $c$ (reaching $b$ with a left turn at time $t=4)$, and a third goes to $(-2,-1)$ with a left turn to $(-2,0)$, and another left turn to reach $c$ at time $t=5$. To return to his start $v_{4}$ again at $t=6$, he would make another left turn at time 6 . These three paths from $v_{4}$ are drawn in Figure 7 . The remaining agent from $v_{4}$ must have gone (dotted lines) to $(-1,2)$ at time $t=3$, and this agent must be the one already met, $\mathcal{E}$. So we can label the paths clockwise to that of $\mathcal{E}$ as $\mathcal{S}, \mathcal{W}$, and $\mathcal{N}$, as in figure 7 . Thus on moves 3 to 6 , the $\mathcal{N}$ agent moves $W, S, E, N$, and since the moves of the $\mathcal{N}$ agent (from any start, since the rotation applied is the identity) are the same as the strategy $g$, we know that the move sequence of $g$ is $(N, S, W, S, E, N, D,-D)$ for some direction $D$ (since there is another SPM of type II at $t=8$ ). To determine the direction $D$, note that since the $\mathcal{N}$ player from $v_{2}$ moves $W, S$ at times $t=3,4$, it was the $\mathcal{E}$ agent who moved $N$ and $W$ (to $(1,0)$ and then to the meeting at $(0,0)$, as drawn in Figure 7 ). Consequently it cannot be the $\mathrm{E}$ agent from $v_{2}$ who moves at $t=7$ to the meeting node $(-1,0)$, which would be a move in direction $D=S$. This leaves the three strategies for II given in $c c 1$ in Figure 5.

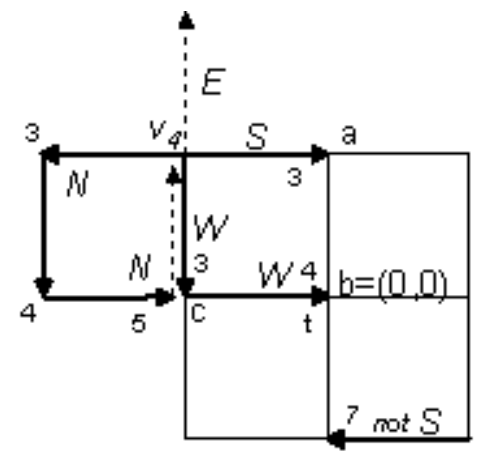

Figure 7: Optimal $g$ for $f$ of $c c 1$

Meeting type [II,I,II,I]: For this meeting type we reverse our usual perspective and adopt the coordinate system of Player II, who is now assumed to start at $(0,0)$, while Player I has 16 agents starting four each at the nodes $v_{i}$. A 
standard optimal strategy $g$ for Player II must begin $N, S$, be at the origin (II's starting node) at times $t=0,2,6$, and visit two starting nodes $a$ and $b$ of I at respective times 4 and 6 . As in the proof of Theorem $21, g$ must be adjacent to the two remaining starting nodes of I at two distinct times. We will determine all $g$ 's with this property, noting that $g$ and its reflection $g^{\prime}$ will be the same in this respect, so we need only consider one of each such pair. We divide the proof into two cases, according to whether $(i)$ nodes $a$ and $b$ are opposite (distance 4) or (ii) on the same side (distance 2) of the square determined by I's starting points. We begin by reducing the possibilities to seven strategies $g_{1}$ to $g_{7}$ (which include the four in Figure 5 as $g_{2}$ to $g_{5}$ ), and their reflections.

(i) By reflectional symmetry may assume that these nodes are $a=(-1,1)$ and $b=(1,-1)$. If $a$ is searched first, then the only path with the required property is $g_{1}=N S W N S E E S$, as drawn in Figure 7. If $b$ is searched first, then the only paths are those given in Figure 5 as $c c 2, c c 3$, and $c c 4$, which we call $g_{2}, g_{3}$, and $g_{4}$, respectively.

(ii) If $a$ and $b$ are both at the top of the square, the two nodes $( \pm 1,1)$, there is no way to be adjacent to both (or even one of) the nodes $( \pm 1,-1)$ twice, as required. If $a$ and $b$ are both on the same (left or right) side, the only path with property (except for its reflection) is the $g$ given in Figure 5 as cc5, which we call $g_{5}$. If $a$ and $b$ are both on the bottom of the square, nodes $( \pm 1,-1)$, then the only potentially optimal paths are $g_{6}$ and $g_{7}$ of Figure 8.

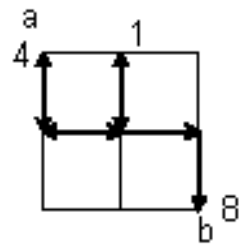

$g_{1}$

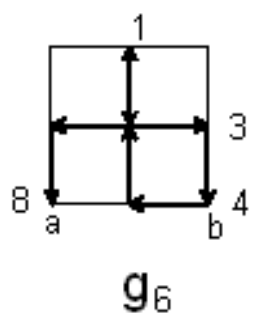

$g_{6}$

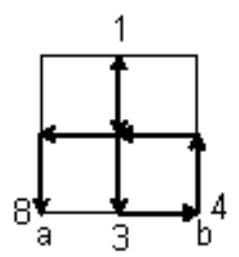

g7

Figure 8: Three $g$ 's to be ruled out

We now exclude all but the four strategies $g$ of $c c 2$ to $c c 5$ from potential optimality by observing that the four agents of every starting node of I unvisited by $g$ must each have one of the meetings with $\mathrm{I}$, and hence no agent can take two of these meetings. Since standard strategies begin $N, E$ for $f$ and $N, S$, for $g$, the same agent of I (namely $\mathcal{E}$ ) from $v_{4}$ is at both meeting nodes $(0,1)$ for $t=1$ and $(0,0)$ for $t=2$, so no $g$ that doesn't visit $v_{4}$ can meet all four agents from $v_{4}$ by time 8 . This argument excludes $g_{6}, g_{7}$ and their reflections, as well as $g_{1}^{\prime}, g_{2}^{\prime}, g_{3}^{\prime}, g_{4}^{\prime}, g_{5}^{\prime}$, from being optimal.

For the rest of the proof we return to our usual perspective where Player I starts at the origin and the agents of Player II start at the 
nodes $v_{i}$, and we denote the strategies $f$ of $c c i$ as $f_{i}, i=2, \ldots, 5$. The strategy $g_{1}$ can be eliminated by observing that the the $\mathcal{W}$ agent of II from $v_{4}$ following $g_{1}$ will not meet Player I paths $f_{2}, f_{3}$, or $f_{4}$; and the $\mathcal{S}$ agent of II from $v_{3}$ following $g_{1}$ will not meet $f_{5}$.

Thus the only potential optimal strategies for Player II in the meeting type [II,I,II,I] are the ones listed in Figure 5 as cc2 to cc5 which we call $g_{2}$ to $g_{5}$. We must show that $g_{i}$ forms an optimal pair only with the corresponding $f_{i}$, $i=2, \ldots, 5$. Observe that the $\mathcal{N}$ agent from $v_{4}$ following $g_{5}$ never meets (up to time 8) $f_{2}, f_{3}$,or $f_{4}$, so such pairings cannot be optimal. Similarly, the $\mathcal{W}$ agent of $v_{2}$ following $g_{4}$ is not met by time 8 by any of the $f_{2}, f_{3}, f_{5}$; and the $\mathcal{E}$ agent of $v_{2}$ following $g_{3}$ is not met by any of the $f_{2}, f_{4}, f_{5}$. Finally the $\mathcal{W}$ agent of $v_{4}$ following $g_{2}$ is not met by either $f_{4}$ or $f_{5}$; and the $\mathcal{N}$ agent of $v_{3}$ is not met by $f_{3}$. Hence for $i=2,3,4,5$, the strategy $g_{i}$ forms an optimal pair only with $f_{i}$, and we are done.

Since we have already established in Corollary 16 that $R\left(\Gamma_{D}\right)=R\left(\Gamma_{D}^{C}\right)$, it follows from Proposition 7 that an optimal strategy pair $(f, g)$ for $\Gamma_{D}^{C}$ is also optimal in the no common clockwise problem $\Gamma_{D}$ if and only if the pair $\left(f, g^{\prime}\right)$ is also optimal in $\Gamma_{D}^{C}$. Since we have a complete list of strategies optimal for $\Gamma_{D}^{C}$ in Figure 5 (according to the previous Theorem), we need only check for which $(f, g)$ in that list the strategy $\left(f, g^{\prime}\right)$ is also in the list. This is easily done. We begin with the Modified Wait For Mommy strategy $c c 6$ with move 7 a $W$. Its reflection $g^{\prime}$ has move 7 in direction $E$, which is not on the list. However all the other strategies in $c c 6$ have their reflections $g^{\prime}$ also in the $c c 6$ group. So all the Modified Wait For Mommy strategies with move 7 being $N$ or $S$ are optimal strategies for the no common clockwise problem. (We note that in Corollary 18.3 of Alpern and Gal (2003) the move 7 of $S$ was missed.) We call these the generalized Anderson-Fekete strategies. It is easily checked that none of the other player II strategies in the list can be reflected (transposing $E$ and $W$ ) and still remain in the list. That is, if a standard optimal strategy $(f, g)$ is not a generalized A-F strategy, then $\left(f, g^{\prime}\right)$ is not optimal in $\Gamma_{D}^{C}$, and hence $(f, g)$ is not optimal in $\Gamma_{D}$. Thus we have established the following characterization of optimal strategies for $\Gamma_{D}$, as a consequence of the previous Theorem.

Corollary 23 A standard form strategy pair is optimal for the 'no common clockwise' rendezvous problem $\Gamma_{D}$ if and only if it is a Modified Wait For Mommy strategy which moves $N$ or $S$ on move 7 (cc6), that is, a generalized Anderson-Fekete strategy.

\section{Planar Rendezvous with Parallel Start}

In this section we consider the parallel start rendezvous problems in the plane, $\Gamma_{P}^{C}$ and $\Gamma_{P}$, where the initial difference vector between the two players is of length 2 and parallel to one of the axes (Definition 1). The starting configurations for the common clockwise formulation of this problem are illustrated in Figures 1 and 2. In Alpern and Gal (2003), a family of strategies called Alter- 
nating Wait For Mommy (AWFM) for this starting configuration and general dimension $n$ is given.

Definition 24 A strategy in $\Gamma_{P}$ is called $A W F M$ if Player I successively visits the $2 n$ possible starting locations of II (in any order) at times $\mathcal{T}_{1}=\{2,6$, $\ldots, 2+4(2 n-1)\}$, while returning to his start $(0,0)$ at the intermediate times $\mathcal{T}_{2}=\{4,8, \ldots, 4(2 n-1)\} ;$ and Player II makes his first move a single unit in any direction, is back at his start times $\mathcal{T}_{1}$, and visits all but one of the possible initial locations of I at times $\mathcal{T}_{2}$. The maximum time for this strategy is clearly $M=2+4(2 n-1)$, or $M=14$ for $n=2$.

The expected meeting times for this strategy family are analyzed in the next section for general (mainly large) $n$. In Alpern and Gal (2003) it was shown that for $n=2$ any AWFM strategy (as shown below in Figure 9) gives the maximal probability of meeting by time $t$ for any $t \leq 7$, and it is suggested that this might in fact be optimal (like the $n=1$ version, where optimality was established by Alpern and Gal (1995)) or even uniformly optimal.

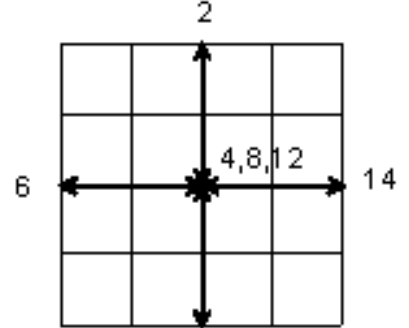

10

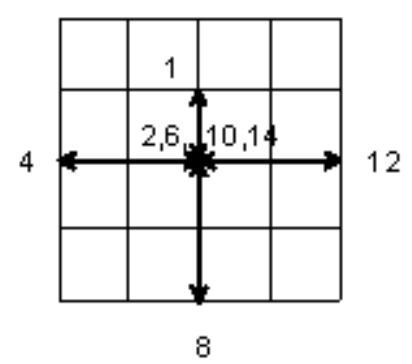

8

$(N, N, S, S, W, W, E, E, S, S, N, N, E, E) \quad(N, S, W, W, E, E, S, S, N, N, E, E, W, W)$

Figure 9: An AWFM strategy $(\breve{f}, \breve{g})$ for $n=2$.

The meeting time sequence $x$ for any $n=2$ version $(\breve{f}, \breve{g})$ of the AWFM in $\Gamma_{P}^{C}$ is given by

$$
\begin{aligned}
(1,3,0,3,0,3,0,2,0,2,0,1,0,1), \text { and since }\left(\breve{f}, \breve{g}^{\prime}\right) \text { is also AWFM, } & \\
T^{C}(\breve{f}, \breve{g}) & =T^{C}\left(\breve{f}, \breve{g}^{\prime}\right)=\frac{99}{16}, \text { and hence also } \\
T(\breve{f}, \breve{g}) & =\frac{99}{16} .
\end{aligned}
$$


However, we have found an alternative strategy with $M=12,(\hat{f}, \hat{g})$ given by

$$
(N, N, S, S, W, W, E, S, E, E, E, N),(N, S, N, N, S, S, S, W, E, E, W, N),
$$

shown in Figure 10, that has a better expected meeting time in $\Gamma_{P}^{C}$, specifically,

$$
T^{C}(\hat{f}, \hat{g})=\frac{97}{16}
$$

Note that its reflection $\left(\hat{f}, \hat{g}^{\prime}\right)$ does not have all its agents met by time 12 , namely the $E$ and $W$ agents from $(0,-2)$. If they were met at time 13 , the expected meeting time of the augmented strategy (of length 13) would be 105/16, so the strategy $(\hat{f}, \hat{g})$ does not preclude the optimality of $(\breve{f}, \breve{g})$ in the no common clockwise problem $\Gamma_{P}$.
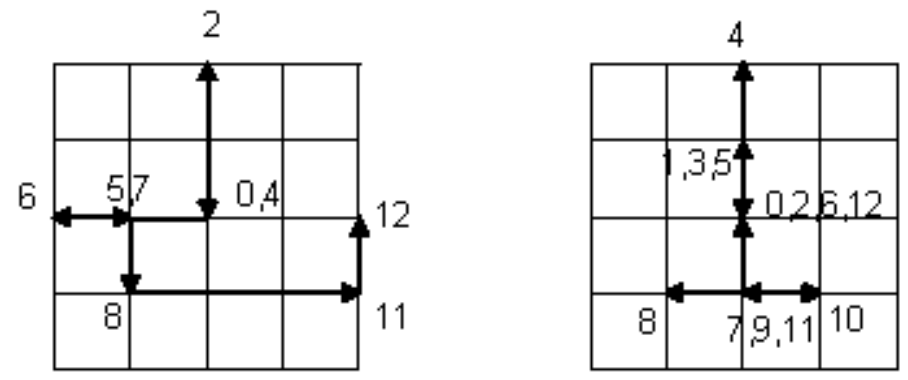

$(N, N, S, S, W, W, E, S, E, E, E, N) \quad(N, S, N, N, S, S, S, W, E, E, W, N)$

Figure 10: The strategy $(\hat{f}, \hat{g})$

However the strategy $(\tilde{f}, \tilde{g})$ drawn in Figure 11 is better than $\mathrm{AWFM}$ in $\Gamma_{P}$. Note that I's strategy $\tilde{f}$ is the same as that for $\operatorname{AWFM}(\breve{f})$ up to time 10 , but then I goes from $(0,-2)$ to $(2,0)$ without going back through his starting point 
$(0,0)$.

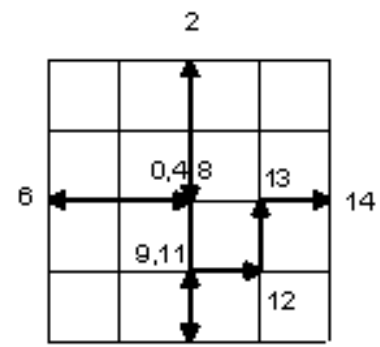

10

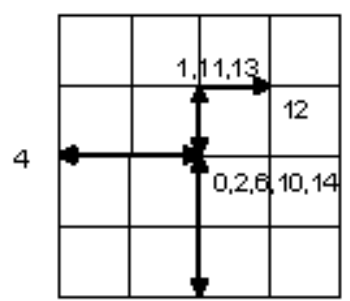

8

$(N, N, S, S, W, W, E, E, S, S, N, E, N, E) \quad(N, S, W, W, E, E, S, S, N, N, N, E, W, S)$

Figure 11: The strategy $(\tilde{f}, \tilde{g})$

We have

$$
\begin{aligned}
T^{C}(\tilde{f}, \tilde{g}) & =\frac{98}{16}, T^{C}\left(\tilde{f}, \tilde{g}^{\prime}\right)=\frac{99}{16}, \text { so } \\
T(\tilde{f}, \tilde{g}) & =\frac{197}{32} .
\end{aligned}
$$

Remark 25 We have shown in Alpern and Baston (2004a) that in fact $(\hat{f}, \hat{g})$ is optimal in the common clockwise problem $\Gamma_{P}^{C}$, which therefore has rendezvous value $R\left(\Gamma_{P}^{C}\right)=97 / 16$, and $(\tilde{f}, \tilde{g})$ is optimal in the no common clockwise problem $\Gamma_{P}$, which therefor has rendezvous value $R\left(\Gamma_{P}\right)=197 / 32$. Hence the parallel start problem is one in which (unlike the diagonal start problem) it does help the players to have a common notion of clockwise. The optimality proofs in both cases are very involved (much more so than for diagonal start).

It is easy to show that

Proposition 26 There are no uniformly optimal strategies in either of the parallel start rendezvous problems $\Gamma_{P}^{C}$ or $\Gamma_{P}$.

Proof. According to Remark 8, it is sufficient to demonstrate that $(\hat{f}, \hat{g})$ is not uniformly optimal in $\Gamma_{P}^{C}$ and that $(\tilde{f}, \tilde{g})$ is not uniformly optimal in $\Gamma_{P}$. To do this we define an additional strategy pair $(\dot{f}, \dot{g})$ given by

$((N, W, S, W, E, E, S, S, N, N, E, E, \ldots),(N, E, W, S, S, S, N, N, W, W, E, E, \ldots))$, 
and calculate the number of agents $y(t)$ met by time $t$ in $\Gamma_{P}^{C}$ for various strategies.

\begin{tabular}{|ccccccccccccccc|}
\hline $\mathrm{y}$ & 1 & 2 & 3 & 4 & 5 & 6 & 7 & 8 & 9 & 10 & 11 & 12 & 13 & 14 \\
\hline$y(\hat{f}, \hat{g})(t)$ & 1 & 4 & 4 & 7 & 7 & 10 & 10 & $11^{*}$ & 12 & 14 & 15 & 16 & & \\
\hline$y(\tilde{f}, \tilde{g})(t)$ & 1 & 4 & 4 & 7 & 7 & 10 & 10 & $12^{*}$ & 12 & 14 & 14 & 15 & 16 & 16 \\
$y\left(\tilde{f}, \tilde{g}^{\prime}\right)(t)$ & 1 & 4 & 4 & 7 & 7 & 10 & 10 & 12 & 12 & 14 & 14 & 15 & 15 & 16 \\
sum above & 2 & 8 & 8 & 14 & 14 & 20 & 20 & 24 & 24 & 28 & 28 & $30^{*}$ & 31 & 32 \\
\hline sum below & 2 & 5 & 6 & 11 & 11 & 17 & 17 & 23 & 23 & 27 & 27 & $31^{*}$ & & \\
$y\left(\dot{f}, \dot{g}^{\prime}\right)(t)$ & 1 & 3 & 3 & 6 & 6 & 9 & 9 & 12 & 12 & 14 & 14 & 16 & \\
$y(\dot{f}, \dot{g})(t)$ & 1 & 2 & 3 & 5 & 5 & 8 & 8 & 11 & 11 & 13 & 13 & 15 & & \\
\hline
\end{tabular}

To see that the optimal strategy $(\hat{f}, \hat{g})$ for $\Gamma_{P}^{C}$ is not uniformly optimal, observe that at time $t=8,11$ agents have been met; while under the strategy $(\tilde{f}, \tilde{g}), 12$ agents have been met. Similarly, under optimal strategy $(\tilde{f}, \tilde{g}), 15$ agents have been met by time $t=12$, and the same number under the reflected strategy $\left(\tilde{f}, \tilde{g}^{\prime}\right)$, or 30 of the 32 agents in the problem $\Gamma_{P}$. However, under the strategy $(\dot{f}, \dot{g}), 15$ agents have been met; under the reflection $\left(\dot{f}, \dot{g}^{\prime}\right) 16$ have been met, for a total of 31 of the 32 agents in the no common clockwise problem $\Gamma_{P}$.

\section{References}

Alpern, S. (1995). The rendezvous search problem. SIAM J. Control \& Optimization 33, 673-683.

Alpern, S. (2002a). Rendezvous search: A personal perspective. Operations Research 50, No. 5, 772-795.

Alpern, S. (2002b). Rendezvous search on labelled graphs. Naval Research Logistics 49, 256-274.

Alpern, S., and Baston, V. (2004a). Rendezvous in higher dimensions. CDAM Research Report Series Centre for Discrete and Applicable Mathematics, LSE 2004-6.

Alpern, S., and Baston, V. (2004b). A common notion of clockwise can help in planar rendezvous. CDAM Research Report Series, Centre for Discrete and Applicable Mathematics, LSE 2004-8.

Alpern, S., and Gal, S. (1995). Rendezvous search on the line with distinguishable players. SIAM J. Control \& Optimization 33, 1270-1276.

Alpern, S., and Gal, S (2003). The theory of search games and rendezvous. International Series in Operations Research and Management Science, Volume 55, Kluwer Academic Publishers, Boston, 336 pp. 
Anderson, E. J., and Essegaier, S. (1995). Rendezvous search on the line with indistinguishable players, SIAM Journal of Control and Optimization 33, 1637-1642.

Anderson, E. J., and Fekete, S. (2001). Two dimensional rendezvous search. Operations Research 49, 107-118.

Baston, V. J. (1999). Two rendezvous search problems on the line, Naval Research Logistics 46, 335-340.

Baston, V. J. and Gal, S. (1998). Rendezvous on the line when the players' initial distance is given by an unknown probability distribution. SIAM Journal of Control and Optimization 36, no. 6, 1880-1889.

Gal, S., (1999). Rendezvous search on the line. Operations Research 47, 974-976.

Howard, J. V. (1999). Rendezvous search on the interval and circle. Operations Research 47, No. 4, 550-558.

Thomas, L. C. and Hulme, P. B. (1997). Searching for targets who want to be found. Journal of the O. R. Society 48, Issue 1, Pages $44-50$. 